Comparação da Composição Centesimal, Mineral e Fitoquímica de Polpas e Cascas de Dez Diferentes Frutas

\author{
Bramont, W. B.; Leal, I. L.; Umsza-Guez, M. A.; Guedes, A. S.; Alves, S. C. \\ O.; Reis, J. H. O.; Barbosa, J. D. V.; Machado, B. A. S.*
}

Rev. Virtual Quim., 2018, 10 (4), 811-823. Data de publicação na Web: 24 de julho de 2018

http://rvq.sbq.org.br

\title{
Comparison of the Centesimal, Mineral and Phytochemical Composition of Pulps and Peel of Ten Different Fruits
}

\begin{abstract}
There are several fruits native to cultivated in Brazil that have not been studied for their antioxidant potential and functional characteristics. This study characterized the physico-chemical composition, mineral and levels of compounds of the pulp and peel of ten fruits. The evaluated fruits were atemoya, ambarella, breadfruit, soursop, jackfruit, lychee, mangaba, sapodilla, hog plum, and sugar apple. Higher protein content was observed (from 1.24 to $3.43 \mathrm{~g} / 100 \mathrm{~g}$ ) in the peel and the pulp in fruits of the Annonaceae family (atemoya, cherimoya and sugar apple), and the sapodilla showed higher lipid content ( 2.37 and $3.37 \mathrm{~g} / 100 \mathrm{~g}$, for pulp and peel respectively). In general, concentration of minerals was higher in the peel than in the pulp. The fruits of the Annonacea family had the highest total phenolic values mainly in the peel. The highest anthocyanin and flavonoids content was found in the peel of lychee, and in the mangaba peel, respectively. Most fruits had higher concentration of the investigated analytes (physicalchemicals, minerals and phytochemicals) in the peels (considered waste from agricultural industries) against their respective pulps.
\end{abstract}

Keywords: phenolics; agribusiness; tropical fruits; flavonoids; anthocyanins; potassium.

\section{Resumo}

Existem várias frutas nativas ou cultivadas no Brasil que ainda não foram estudadas quanto ao seu potencial antioxidante e funcionalidade. Neste estudo foi caracterizada a composição físico-química, mineral e de compostos bioativos da polpa e casca de dez frutas. As frutas avaliadas foram atemoia, cajarana, fruta-pão, graviola, jaca, lichia, mangaba, sapoti, seriguela e pinha. Foi verificado maior teor de proteína $(1,24$ a 3,43g/100g) nas frutas da família Annonaceae (atemoia, graviola e pinha) tanto na casca como na polpa, e, teores de lipídeos mais altos para o sapoti (polpa e casca: 2,37 e 3,37g/100g, respectivamente). De forma geral, a concentração dos minerais nas cascas das frutas foi superior quando comparada às polpas. As frutas da família Annonaceae apresentaram os maiores valores de fenólicos, principalmente na casca. Os maiores teores de antocianinas e flavonoides foram verificados nas cascas da lichia e da mangaba, respectivamente. A maioria das frutas apresentou maior concentração dos parâmetros investigados (físico-químicos, minerais e fitoquímicos) nas cascas (consideradas resíduos agroindustriais) quando comparada com as respectivas polpas.

Palavras-chave: compostos fenólicos; agroindústria; frutas tropicais; flavonoides; antocianinas; potássio.

\footnotetext{
* Centro Universitário SENAI CIMATEC, Serviço Nacional de Aprendizagem Industrial, CEP 41650-010, Salvador-BA, Brasil.

$M$ brunam@fieb.org.br DOI: $10.21577 / 1984-6835.20180059$
} 


\title{
Comparação da Composição Centesimal, Mineral e Fitoquímica de Polpas e Cascas de Dez Diferentes Frutas
}

\author{
Wagner B. Bramont, ${ }^{\mathrm{a}}$ Ingrid L. Leal, ${ }^{\mathrm{a}}$ Marcelo Andrés Umsza-Guez, ${ }^{\mathrm{b}}$
} Alessandra da S. Guedes, ${ }^{c}$ Sheila Cristina de O. Alves, ${ }^{d}$ João Henrique de

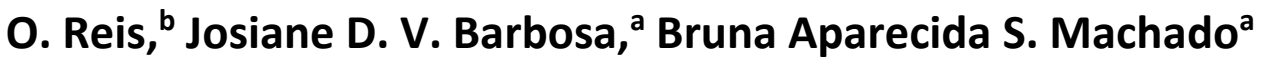

a Centro Universitário SENAI CIMATEC, Serviço Nacional de Aprendizagem Industrial, CEP 41650-010, Salvador-BA, Brasil.

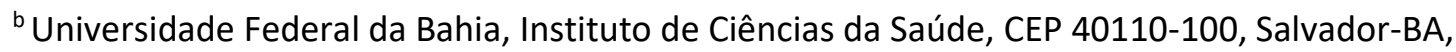
Brasil.

' União Metropolitana de Educação e Cultura, CEP 41194-020, Salvador-BA, Brasil.

dUniversidade Estadual de Campinas, Faculdade de Engenharia de Alimentos, CEP 13083-862, Campinas-SP, Brasil.

* brunam@fieb.org.br

Recebido em 2 de março de 2017. Aceito para publicação em 5 de julho de 2018

\section{Introdução}

\section{Material e métodos}

2.1. Determinação da composição centesimal

2.2. Extração e quantificação dos Compostos Fenólicos Totais, Flavonoides e Antocianinas

2.3. Avaliação estatística

\section{Resultados e discussão}

\section{Conclusão}

\section{Introdução}

O Brasil, país detentor de uma vasta biodiversidade e grande extensão territorial, possui diferentes espécies nativas de frutas ainda pouco comerciais, mas que tem grande importância socioeconômica e enorme potencial de exploração comercial. No entanto, existem várias frutas nativas e/ou cultivadas no Brasil que ainda não foram estudadas quanto ao seu potencial antioxidante, como também outras capacidades funcionais e nutricionais. ${ }^{1,2,3,4}$ Além disso, o país se destaca como o terceiro maior produtor mundial de frutas, que supera os 41 milhões de toneladas, perdendo apenas para China e Índia. ${ }^{5}$. Diferentes estudos apontam os benefícios associados ao consumo regular de frutas, principalmente relacionada à característica antioxidante das mesmas devido ao seu 
conteúdo de vitaminas e compostos fenólicos. Septembre-Malaterre et al. (2016), ${ }^{6}$ mostraram a relação positiva da prevenção da obesidade com as propriedades nutricionais e antioxidantes de frutas tropicais: como a banana, lichia, manga, maracujá e abacaxi, cultivadas na ilha de Réunion (território francês).

Os efeitos bioquímicos e farmacológicos destes fitoquímicos são muito vastos, destacando-se várias ações como a antioxidante, anti-inflamatória, antiplaquetária, antialérgicos, inibidores de enzimas como a prostaglandina sintetase, a lipoxigenase e a ciclo-oxigenase. Quando naturalmente presentes nos alimentos, flavonoides são antioxidantes que evitam a formação de radicais livres, reduzindo a necessidade de consumo da vitamina $C$ suplementada. ${ }^{7}$ Além das suas propriedades biológicas, os compostos antioxidantes naturais são também de interesse na indústria de cosméticos, farmacêutica e especialmente nas indústrias alimentares, uma vez que também podem ser utilizados substituindo os sintéticos, proporcionando uma proteção significativa contra a degradação oxidativas. ${ }^{8}$ É bem conhecida a importância das frutas e vegetais como fonte de minerais para a saúde em várias funções fisiológicas. Pesquisadores verificaram o declínio histórico na concentração de minerais presentes nesses vegetais, o que torna fundamental o estudo dos mesmos de forma integral para o melhor aproveitamento. ${ }^{9}$

Destaca-se que, frutas não produzidas de forma comercial, são geralmente obtidas por extrativismo (ex. jaca, fruta-pão, cajarana, entre outras) e precisam ser melhor estudadas em relação ao seu potencial funcional. A inserção integral das frutas cascas $^{10}$ e polpa ${ }^{2}$ - na dieta da população apresenta inúmeras vantagens nutricionais e de benefícios à saúde, como já confirmadas em pesquisas. Muitas frutas utilizadas para a elaboração de produtos (desidratados, sucos, geleias, néctares, compotas, entre outros) os principais subprodutos da industrialização são a casca e a semente, que na maioria dos casos são descartadas. Neste contexto, a agroindústria de beneficiamento de frutas gera resíduos que variam de 15 até $50 \%$ do total da fruta processada. ${ }^{11}$ Estes subprodutos podem ser convertidos em farinha, utilizando métodos de desidratação e trituração e posteriormente junto com farinha de trigo ser incorporada na formulação de bolos, biscoitos e pães, enriquecendo os produtos elaborados com fibras, compostos bioativos, minerais, entre outros. ${ }^{12}$ Por este motivo, aumentaram as pesquisas referentes ao aproveitamento de subprodutos do processamento de frutas e sua aplicação em diferentes produtos e consequentemente, proporcionam um incremento e aumento das características funcionais do alimento desenvolvido. ${ }^{13,14}$

De acordo com vários estudos, o conteúdo de compostos fenólicos totais e de flavonoides, bem como a atividade antioxidante é particularmente elevado na casca de algumas frutas, e em muito dos casos, superior ao da polpa. ${ }^{2,15,16}$

Neste contexto, a indústria de alimentos vem inovando nos produtos e processos, onde as características nutracêuticas/funcionais dos alimentos são conservados/aprimorados na expectativa de atender um púbico mais exigente, informado e consciente. $\mathrm{Na}$ perspectiva de melhoria deste panorama, pesquisas científicas junto às indústrias do setor alimentício tornam-se necessária no desenvolvimento de novos produtos com maior valor nutricional agregado, utilizando em sua escala produtiva os resíduos agroindustriais, como por exemplo, as cascas, caroço, sementes e bagaço de frutas. ${ }^{17-21}$

É importante destacar que o Brasil é um dos países que mais produz resíduos agroindustriais, e, no ano de 2009 foram 290 milhões de toneladas, segundo o Plano Nacional de Resíduos Sólidos (2011) ${ }^{22}$, entre eles subprodutos das indústrias de polpas de frutas, o que tem contribuído para o aumento do volume de lixo orgânico, provocando problemas ambientais. ${ }^{23}$

Com base no contexto apresentado, o objetivo deste estudo foi realizara comparação da composição centesimal, mineral e fitoquímica de polpas e cascas de 
dez diferentes frutas cultivadas no Brasil.

\section{Material e métodos}

As cascas e polpas de frutas avaliadas neste estudo foram: atemoia (Annona atemoya), cajarana (Spondias lutea L.), fruta-pão (Artocarpus incisa L.), graviola (Annona muricata L.), jaca (Artocarpus integrifólia L), lichia (Litchi chinensia), mangaba (Hancornia speciosa), sapoti (Manilkara zapota L.), seriguela (Spondias purpúrea) e pinha (Annona squamosa L.).

Aproximadamente 1 quilo de cada fruta foi obtida no comércio local da cidade de Salvador (Bahia - Brasil). O material foi levado ao Laboratório de Bromatologia, no Senai Dendezeiros, onde procedeu-se toda caracterização. Foram lavadas com água clorada (100ppm), retiradas manualmente as cascas de cada fruta e as polpas separadas das sementes. As cascas e as polpas foram trituradas separadamente no multiprocessador de alimentos, mantidas congeladas a $-18^{\circ} \mathrm{C}$ em sacos plásticos. Todas as análises foram realizadas em triplicata.

\subsection{Determinação da composição centesimal}

A umidade e o teor de cinzas foram determinados seguindo o método gravimétrico da Association of Official Analytical (AOAC, 1997). ${ }^{24} \mathrm{O}$ teor de proteína bruta foi determinado com base na metodologia de Kjeldahl-Micro, ${ }^{25}$ calculada em função dos teores de nitrogênio total multiplicado pelo fator de 6,25. Os lipídeos totais foram extraídos e quantificados por Soxhlet, segundo método da International Union of Pure and Applied Chemistry (IUPAC, 1979). ${ }^{26}$ Os carboidratos foram determinados por diferença do somatório dos outros componentes, subtraindo-se a média encontrada por 100. Os teores de sódio $\left(\mathrm{Na}^{+}\right)$, potássio $\left(\mathrm{K}^{+}\right)$, lítio $\left(\mathrm{Li}^{+}\right)$e cálcio $\left(\mathrm{Ca}^{2+}\right)$ foram analisados em fotômetro de chamas digital (Digmed) seguindo o método do Instituto Adolfo Lutz a partir das cinzas brutas. ${ }^{27}$

2.2. Extração e quantificação dos Compostos Fenólicos Totais, Flavonoides e Antocianinas - Os extratos das cascas e polpas das diferentes frutas para determinação dos compostos fenólicos foram obtidos conforme metodologia proposta por Larrauri et al. (1997). ${ }^{28}$ Os extratos foram preparados com a adição sequencial de $40 \mathrm{ml}$ de solução metanol:água (50:50, v:v) e $40 \mathrm{ml}$ de solução acetona:água (70:30, v:v) em $5 \mathrm{~g}$ de amostra, à temperatura ambiente durante 60 minutos. Após centrifugação a $2500 \mathrm{rpm}$ durante 15 minutos, os sobrenadantes foram combinados e aferidos até $100 \mathrm{ml}$ com água destilada. Os extratos foram armazenados em freezer a $-18^{\circ} \mathrm{C}$ até o momento da realização da análise. A determinação dos compostos fenólicos foi realizada por espectrofotometria por meio do método de Folin-Ciocalteau, de acordo com procedimento de Obanda \& Owuor (1997), ${ }^{29}$ expressos em miligramas (mg) de equivalentes ácido gálico (EAG) por $100 \mathrm{~g}$ de amostra.

As análises de antocianinas e flavonoides totais foram realizadas segundo Francis (1982). ${ }^{30}$ A solução extratora utilizada foi etanol $95 \%$ em $\mathrm{HCl} 1,5 \mathrm{~N}(85: 15, \mathrm{v} / \mathrm{v})$, a absorbância das amostras foi lida a $535 \mathrm{~nm}$ e $374 \mathrm{~nm}$ para quantificação das antocianinas e flavonoides, respectivamente. Os resultados foram expressos em miligramas ( $\mathrm{mg}$ ) de equivalentes de quercetina (EQ)/100g de amostra, onde foi utilizado o coeficiente de extinção da quercetina para expressar os resultados.

\subsection{Avaliação estatística}

Os resultados foram submetidos à análise de variância (ANOVA) e as médias das triplicatas foram comparadas pelo Teste de Scott-Knott ao nível de 95\% de significância, utilizando o software Assistat 7.7. 


\section{Resultados e discussão}

$\mathrm{Na}$ Tabela 1 encontram-se os valores médios da composição centesimal, realizada nas frações da polpa e casca de dez diferentes frutas. De forma geral, o teor de umidade apresentou-se maior nas polpas das frutas quando relacionado às respectivas cascas, exceto para a jaca e fruta-pão. A cajarana apresentou maior teor de umidade tanto na casca quanto na polpa. Canuto et al. (2010), ${ }^{31}$ verificaram um valor de $96,1 \%$ de umidade em polpa da cajarana.

Destaca-se que o conteúdo de umidade nas frutas está diretamente associado à sua perecibilidade, levando as indústrias a adotarem processos tecnológicos de conservação como a desidratação convencional, desidratação osmótica, liofilização, entre outros, reduzindo seu percentual de perdas. ${ }^{32,33}$

A variação no teor de umidade para casca e polpa, respectivamente, da graviola $(80,30 \mathrm{e}$ $65,95 \%)$, pinha $(66,50$ e $53,4 \%)$ e atemoia $(65,85$ e $56,55 \%)$ - híbrido resultante do cruzamento da cherimóia (Annona cherimola mill) com a fruta-do-conde; assemelham-se aos resultados obtidos por Marcellini et al. (2003).$^{34} \mathrm{O}$ teor de água determinado para a polpa do sapoti e a polpa e cascada seriguela, corroboram com os valores apresentados por Sousa et al. (2012) ${ }^{23}$ e Lima et al. (2009), ${ }^{35}$ para as mesmas frutas.

Foi verificada que atemoia (casca e polpa) apresentou valor superior às outras amostras em relação ao percentual proteico, entretanto a quantidade de proteína encontrada na casca $(3,43 \%)$ foi inferior ao apresentado por Cruz et al. (2013) ${ }^{36} \mathrm{~A}$ seriguela apresentou um dos maiores teores de proteínas, lipídeos e cinzas na casca em relação à sua polpa, corroborando com os valores verificados por Lima et al. (2009). ${ }^{35}$
O teor de lipídeos apresentou-se maior nas cascas das frutas. De acordo com Monteiro et al. (2009), ${ }^{37}$ os lipídeos compreendem menos de $1 \%$ da maioria dos frutos e hortaliças, confirmando os resultados apresentados paras as polpas na Tabela 1, com exceção do sapoti e mangaba. Essa última difere-se do conteúdo encontrado por Silva et al., (2008). ${ }^{38}$

É importante destacar que o baixo teor lipídico determinado para fruta-pão encontrado neste estudo (característico da maioria das frutas) está de acordo com o apresentado na Tabela Brasileira de Composição de Alimentos para esta fruta, ${ }^{39}$ com $0,2 \%$ (fruta-pão). Por ser uma fruta rica em calorias $(115,88 \mathrm{Kcal})$, carboidratos $(27,40 \%)$, água, e apresentar baixo teor de gorduras $(0,24 \%)$, pode ser aproveitada tanto in natura, como em produtos processados, por exemplo em: fruta seca, farinha panificável e para extração de amido. ${ }^{40}$

A composição centesimal da lichia apresentada por Motta et al. (2009) ${ }^{41}$ está próxima ao observado (Tabela 1), com variações para os parâmetros de umidade, carboidratos e valor calórico total. O diferencial no teor de carboidratos pode estar associado ao percentual de fibra alimentar.

Em relação às cinzas, a maior proporção foi observada nas cascas de todas as frutas com exceção da jaca e da mangaba, que apresentaram maior concentração na polpa. O teor de cinzas nas cascas de graviola, jaca e lichia não apresentaram diferença estatística entre si $(p>0,05)$. Os valores de cinzas avaliados na casca da atemoia foram superiores aos da polpa em 43,93\% (Tabela 1). Esses valores são semelhantes aos encontrados por Morzelle et al. $(2015)^{3} \mathrm{em}$ frutas do cerrado brasileiro. Destaca-se que a variação no teor de cinzas e minerais entre as frutas pode estar relacionada com o cultivar, o grau de maturação, a origem geográfica e o tipo e a fertilidade do solo (Rizzon et al., $2005)^{42}$, bem como, ao método empregado para a análise. 
Tabela 1. Composição centesimal em base úmida $(\mathrm{g} / 100 \mathrm{~g})$ das cascas e polpas de dez diferentes frutas

\begin{tabular}{|c|c|c|c|c|c|c|c|c|c|c|c|c|}
\hline \multirow[t]{2}{*}{ Frutas } & \multicolumn{2}{|c|}{ Umidade } & \multicolumn{2}{|c|}{ Proteína } & \multicolumn{2}{|c|}{ Cinzas } & \multicolumn{2}{|c|}{ Lipídeos } & \multicolumn{2}{|c|}{ Carboidratos* } & \multicolumn{2}{|c|}{$\mathrm{VCT}^{* *}$} \\
\hline & Casca & Polpa & Casca & Polpa & Casca & Polpa & Casca & Polpa & Casca & Polpa & Casca & Polpa \\
\hline Atemoia & $56,55 \pm 0,35^{8}$ & $65,85 \pm 1,48^{f}$ & $3,43 \pm 0,05^{a}$ & $1,41 \pm 0,05^{a}$ & $1,98 \pm 0,02^{\mathrm{a}}$ & $1,11 \pm 0,02^{b}$ & $1,93 \pm 0,02^{d}$ & $0,29 \pm 0,02^{g}$ & 36,11 & 31,34 & 175,53 & 133,61 \\
\hline Cajarana & $81,30 \pm 1,41^{a}$ & $85,00 \pm 0,42^{a}$ & $0,54 \pm 0,04^{f}$ & $1,12 \pm 0,06^{d}$ & $0,61 \pm 0,02^{\mathrm{g}}$ & $0,42 \pm 0,03^{g}$ & $1,18 \pm 0,52^{e}$ & $0,33 \pm 0,10^{f}$ & 16,37 & 13,13 & 78,26 & 59,97 \\
\hline Fruta-pão & $73,05 \pm 0,07^{c}$ & $70,50 \pm 0,28^{d}$ & $0,96 \pm 0,20^{d}$ & $1,03 \pm 0,31^{e}$ & $1,19 \pm 0,01^{d}$ & $0,83 \pm 0,02^{c}$ & $1,27 \pm 0,62^{e}$ & $0,24 \pm 0,01^{i}$ & 23,53 & 27,40 & 109,39 & 115,88 \\
\hline Graviola & $65,95 \pm 0,63^{e}$ & $80,30 \pm 1,83^{b}$ & $1,62 \pm 0,04^{c}$ & $1,37 \pm 0,09^{b}$ & $0,98 \pm 0,03^{e}$ & $0,56 \pm 0,06^{e}$ & $0,80 \pm 0,098$ & $0,71 \pm 0,19^{e}$ & 30,65 & 17,06 & 136,28 & 80,11 \\
\hline Jaca & $64,50 \pm 0,14^{f}$ & $46,60 \pm 0,42^{8}$ & $0,26 \pm 0,03^{8}$ & $1,25 \pm 0,19^{c}$ & $1,03 \pm 0,02^{e}$ & $1,32 \pm 0,14^{a}$ & $0,98 \pm 0,16^{f}$ & $0,28 \pm 0,07^{h}$ & 33,23 & 50,55 & 142,78 & 209,72 \\
\hline Lichia & $70,35 \pm 2,19^{d}$ & $80,60 \pm 0,56^{b}$ & $1,0 \pm 0,12^{d}$ & $0,86 \pm 0,04^{f}$ & $1,02 \pm 0,01^{e}$ & $0,41 \pm 0,03^{g}$ & $2,10 \pm 0,14^{c}$ & $0,79 \pm 0,08^{c}$ & 25,53 & 17,34 & 125,02 & 79,91 \\
\hline Mangaba & $52,60 \pm 2,54$ & $76,10 \pm 0,14^{c}$ & $0,78 \pm 0,09^{e}$ & $0,79 \pm 0,15^{g}$ & $0,43 \pm 0,02^{\mathrm{h}}$ & $0,50 \pm 0,13^{f}$ & $3,59 \pm 0,35^{a}$ & $2,57 \pm 0,05^{a}$ & 42,60 & 20,04 & 205,83 & 106,45 \\
\hline Sapoti & $73,70 \pm 1,55^{b}$ & $78,65 \pm 0,35^{b}$ & $0,41 \pm 0,18^{f}$ & $0,89 \pm 0,06^{f}$ & $1,32 \pm 0,10^{c}$ & $0,52 \pm 0,01^{f}$ & $3,47 \pm 0,09^{b}$ & $2,37 \pm 0,07^{b}$ & 21,10 & 17,57 & 117,27 & 95,17 \\
\hline Seriguela & $66,15 \pm 1,48^{e}$ & $79,25 \pm 0,07^{b}$ & $0,73 \pm 0,09^{e}$ & $0,47 \pm 0,28^{h}$ & $0,90 \pm 0,06^{f}$ & $0,40 \pm 0,28^{g}$ & $1,25 \pm 0,05^{e}$ & $0,76 \pm 0,02^{d}$ & 30,97 & 19,12 & 290,29 & 85,20 \\
\hline Pinha & $53,4 \pm 3,39^{h}$ & $66,50 \pm 0,84^{e}$ & $2,86 \pm 0,17^{b}$ & $1,24 \pm 0,19^{c}$ & $1,40 \pm 0,02^{b}$ & $0,63 \pm 0,04^{d}$ & $1,06 \pm 0,07^{f}$ & $0,27 \pm 0,06^{h}$ & 41,28 & 31,36 & 186,10 & 132,83 \\
\hline
\end{tabular}

Dados apresentados como média \pm desvio padrão de três replicatas/amostra; Valores na mesma coluna que apresentam a mesma letra não apresentam diferenças significativas $(p>0,05)$ pelo o Teste de Scott-Knott a 95\% de confiança.

*Carboidratos expresso sem a determinação do teor de fibras totais;

${ }^{* *}$ Valor Calórico Total.

resultados da determinação dos minerais avaliados estão apresentados na Tabela 2. A casca e a polpa do sapoti apresentaram maiores teores de sódio que as frutas restantes. A porção não comestível da jaca e a polpa da mangaba apresentaram os menores valores para esse micronutriente.

De forma geral, os outros minerais apresentaram-se em concentrações superiores nas cascas, somente a polpa da seriguela foi superior que a casca em teor de potássio e lítio. A atemoia foi a fruta que apresentou maiores valores de potássio e lítio para ambas porções (casca e polpa) estudadas. $O$ sapoti apresentou maior teor de cálcio nas duas porções estudadas. $\mathrm{O}$ cálcio, importante para os tecidos ósseos, foi encontrando em maior quantidade nas cascas avaliadas. Assim, as cascas podem ser consideradas como um importante ingrediente para ser incorporado em novos alimentos, tendo em vista ser uma fonte de minerais, que por sua vez, exercem várias funções fisiológicas benéficas no organismo. ${ }^{43}$

Dentre as dez frutas estudadas, a atemoia apresentou a maior concentração de potássio, tanto na casca $(867,41 \mathrm{mg} / 100 \mathrm{~g})$ quanto na polpa $(399,44 \mathrm{mg} / 100 \mathrm{~g})$. De acordo com a VI Diretrizes Brasileiras de Hipertensão, ${ }^{44}$ os benefícios sobre a pressão arterial têm sido associados ao alto consumo de potássio. Como essa fruta apresentou um elevado teor de potássio, e ainda, baixo teor de sódio $(5,86$ $\mathrm{mg} / 100 \mathrm{~g}$ na polpa), pode-se avaliar a inclusão da mesma em diferentes alimentos utilizados para suplementação de potássio com fins terapêuticos no controle da pressão arterial. 
Tabela 2. Composição do teor de minerais $(\mathrm{mg} / 100 \mathrm{~g})$ das cascas e polpas de dez frutas.

\begin{tabular}{|c|c|c|c|c|c|c|c|c|}
\hline \multirow{2}{*}{ Frutas } & \multicolumn{2}{|c|}{ Sódio } & \multicolumn{2}{|c|}{ Potássio } & \multicolumn{2}{|c|}{ Lítio } & \multicolumn{2}{|c|}{ Cálcio } \\
\hline & Casca & Polpa & Casca & Polpa & Casca & Polpa & Casca & Polpa \\
\hline Atemoia & $2,91 \pm 0,73^{e}$ & $5,86 \pm 1,91^{d}$ & $867,41 \pm 8,59^{a}$ & $399,44 \pm 14,43^{a}$ & $2,91 \pm 0,43^{a}$ & $2,46 \pm 1,54^{a}$ & $18,95 \pm 1,00^{c}$ & $9,40 \pm 0,70^{b}$ \\
\hline Cajarana & $2,85 \pm 1,13^{e}$ & $3,30 \pm 0,31^{f}$ & $152,26 \pm 0,75^{h}$ & $106,13 \pm 0,91^{g}$ & $1,28 \pm 0,57 \mathrm{~g}$ & $0,45 \pm 0,07^{f}$ & $18,98 \pm 2,13^{c}$ & $2,82 \pm 0,59^{d}$ \\
\hline Fruta-pão & $3,15 \pm 1,57^{e}$ & $5,98 \pm 1,02^{d}$ & $410,65 \pm 3,71^{c}$ & $271,03 \pm 8,00^{c}$ & $1,65 \pm 0,93^{e}$ & $0,72 \pm 0,01^{e}$ & $6,18 \pm 3,02^{\mathrm{g}}$ & $3,13 \pm 1,15^{d}$ \\
\hline Graviola & $3,11 \pm 0,28^{e}$ & $3,43 \pm 0,28^{e}$ & $335,16 \pm 6,44^{f}$ & $9,51 \pm 3,85^{i}$ & $1,29 \pm 0,31^{\mathrm{g}}$ & $0,85 \pm 0,25^{d}$ & $9,41 \pm 0,38^{f}$ & $6,01 \pm 0,71^{c}$ \\
\hline Jaca & $1,65 \pm 0,07^{f}$ & $0,77 \pm 0,17^{h}$ & $393,54 \pm 12,20^{d}$ & $369,39 \pm 11,60^{b}$ & $1,34 \pm 0,35^{f}$ & $0,79 \pm 0,08^{d}$ & $13,71 \pm 1,74^{e}$ & $0,76 \pm 0,13^{f}$ \\
\hline Lichia & $4,25 \pm 0,43^{d}$ & $6,60 \pm 0,55^{b}$ & $220,95 \pm 0,18^{g}$ & $143,60 \pm 6,32^{f}$ & $2,36 \pm 0,45^{b}$ & $0,73 \pm 0,03^{e}$ & $59,92 \pm 0,52^{b}$ & $2,75 \pm 1,95^{d}$ \\
\hline Mangaba & $6,88 \pm 1,33^{c}$ & $0,35 \pm 0,11^{i}$ & $139,89 \pm 3,17^{\mathrm{h}}$ & $4,83 \pm 1,77^{j}$ & $1,08 \pm 0,17^{h}$ & $0,26 \pm 0,05^{g}$ & $4,04 \pm 0,95^{h}$ & $1,08 \pm 0,23^{f}$ \\
\hline Sapoti & $23,46 \pm 1,13^{a}$ & $8,61 \pm 1,73^{a}$ & $366,00 \pm 0,71^{e}$ & $104,86 \pm 0,44^{h}$ & $1,79 \pm 0,60^{d}$ & $0,94 \pm 0,33^{c}$ & $71,73 \pm 1,79^{a}$ & $14,06 \pm 1,89^{a}$ \\
\hline Seriguela & $2,45 \pm 0,96^{e}$ & $2,41 \pm 0,93^{g}$ & $8,45 \pm 0,10^{i}$ & $150,09 \pm 3,17^{e}$ & $1,37 \pm 0,56^{f}$ & $1,76 \pm 0,22^{b}$ & $6,78 \pm 1,71^{\mathrm{g}}$ & $0,35 \pm 0,34^{g}$ \\
\hline Pinha & $9,94 \pm 0,36^{b}$ & $6,04 \pm 1,16^{c}$ & $518,88 \pm 3,09^{b}$ & $238,62 \pm 4,69^{d}$ & $1,94 \pm 0,14^{c}$ & $0,77 \pm 0,39^{e}$ & $14,72 \pm 1,20^{d}$ & $2,12 \pm 0,83^{e}$ \\
\hline
\end{tabular}

Dados apresentados como média \pm desvio padrão de três replicatas/amostra; Valores na mesma coluna que apresentam a mesma letra não apresentam diferenças significativas $(p>0,05)$ pelo Teste de Scott-Knotta 95\% de confiança.

Pesquisas vêm demonstrando que frutas de biomas poucos explorados ou de frutas não comercialmente produzidas de regiões tropicais e subtropicais necessitam de mais estudos para aprofundar os conhecimentos relacionados às suas características nutricionais e funcionais, com o objetivo de incentivar o consumo das mesmas pela população. ${ }^{1,45,46}$

Ressalta-se que os subprodutos agroindustriais cuja matéria-prima é a fruta têm sido aplicados no melhoramento de alimentos ou na inovação de novos produtos, como por exemplo, seu uso na produção de biscoito, ${ }^{47,48,49}$ geleia, ${ }^{50,10}$ sobremesa, ${ }^{51}$ barra de cereais, ${ }^{18}$ suco de maçã processado com casca de arroz, ${ }^{52}$ farinha de casca de batata inglesa na elaboração de pão integral, ${ }^{53}$ farinha de pupunha (Bactris gasipaes Kunth) como um novo ingrediente alimentar, ${ }^{54}$ entre outros.

Populações cuja dieta é rica em compostos fenólicos de origem vegetal (cereais, frutas e vegetais) vêm mostrando através de estudos clínicos e epidemiológicos que este tipo de alimentação é um dos principais fatores que contribuem para expressiva redução nos casos de doenças crônico-degenerativas. ${ }^{55-57}$ A caracterização fitoquímica das dez frutas está apresentada na Tabela 3.

Neste estudo, a quantificação do teor de flavonoides totais ( $\mathrm{mg}$ de quercetina/g de amostra) foi superior nas cascas das amostras analisadas, quando comparadas com o conteúdo nas polpas, as quais são utilizadas comumente na alimentação humana. Rocha et al. (2015) encontraram valores inferiores de flavonoides para as frutas do cerrado (cagaita, chichá, cajuí e macaúba). Destaca-se que a variação na concentração de flavonoides nas frutas pode ser influenciada por fatores como estação do período sazonal, incidência de radiação UV, clima, composição do solo, preparo e processamento do alimento. ${ }^{58}$

A cor de um alimento é um importante fator de escolha pelo consumidor. No caso das frutas, tem-se estudado a estabilidade das antocianinas como corantes naturais associados às suas propriedades antioxidantes, resultando num produto final com valor agregado à sua imagem, além de suas propriedades funcionais apresentando características benéficas para a saúde do consumidor, por possuírem capacidade protetora contra o estresse oxidativo, atuando na prevenção e/ou redução do risco de desenvolvimento de doenças do coração, alguns tipos de carcinomas e outras doenças, e também, além da sua capacidade de inativar radicais livres..$^{59,60}$ Foi identificada uma concentração de antocianinas superior em todas as cascas, com exceção da jaca (Tabela 3).

Rev. Virtual Quim. |Vol 10| |No.4| |811-823| 
Os maiores teores de antocianinas (mg de quercetina/g) foram encontrados nas cascas de lichia $(3,86)$, pinha $(3,65)$, atemoia $(3,24)$ e mangaba $(3,17)$, por outro lado, a maior concentração de antocianina em polpa foi verificada na mangaba, com $1,46 \mathrm{mg}$ de quercetina/g. Rocha et al. $(2015)^{57}$ determinaram valores similares de antocianinas nas frutas do cerrado estudadas, sendo que o jatobá apresentou a maior concentração de antocianinas dentre as frutas avaliadas.

Tabela 3. Caracterização fitoquímica das cascas e polpas de dez frutas

\begin{tabular}{|c|c|c|c|c|c|c|}
\hline \multirow[t]{2}{*}{ Frutas } & \multicolumn{2}{|c|}{$\begin{array}{l}\text { Flavonoides mg } \\
\text { EQ/100g }\end{array}$} & \multicolumn{2}{|c|}{$\begin{array}{c}\text { Antocianinas mg } \\
\mathrm{EQ} / 100 \mathrm{~g}\end{array}$} & \multicolumn{2}{|c|}{ Fenólicos totais mg EAG/100g } \\
\hline & Casca & Polpa & Casca & Polpa & Casca & Polpa \\
\hline Atemoia & $10,4 \pm 0,04^{d}$ & $1,2 \pm 0,01^{c}$ & $3,24 \pm 0,02^{c}$ & $0,53 \pm 0,01^{c}$ & $5506,78 \pm 0,52 a$ & $290,62 \pm 0,36^{h}$ \\
\hline Cajarana & $4,8 \pm 0,02^{g}$ & $1,1 \pm 0,01^{c}$ & $0,96 \pm 0,02^{g}$ & $0,32 \pm 0,01^{f}$ & $718,61 \pm 0,10^{f}$ & $617,99 \pm 0,25^{\mathrm{e}}$ \\
\hline Fruta-pão & $3,9 \pm 0,02^{h}$ & $1,1 \pm 0,01^{c}$ & $0,43 \pm 0,01^{h}$ & $0,23 \pm 0,01^{g}$ & $325,03 \pm 0,84^{\mathrm{g}}$ & $137,44 \pm 0,31^{i}$ \\
\hline Graviola & $7,1 \pm 0,02^{f}$ & $1,2 \pm 0,02^{c}$ & $1,43 \pm 0,02^{f}$ & $0,65 \pm 0,01^{b}$ & $2458,18 \pm 0,29^{c}$ & $1300,18 \pm 0,12^{a}$ \\
\hline Jaca & $1,8 \pm 0,02^{j}$ & $1,4 \pm 0,01^{c}$ & $0,24 \pm 0,02^{i}$ & $0,38 \pm 0,02^{\mathrm{e}}$ & $191,10 \pm 0,01^{\mathrm{g}}$ & $69,60 \pm 0,01^{j}$ \\
\hline Lichia & $8,1 \pm 0,02^{\mathrm{e}}$ & $1,1 \pm 0,01^{c}$ & $3,86 \pm 0,02$ a & $0,18 \pm 0,01^{\mathrm{h}}$ & $658,99 \pm 0,18^{f}$ & $501,59 \pm 0,24^{g}$ \\
\hline Mangaba & $18,6 \pm 0,03^{a}$ & $2,6 \pm 0,01^{\underline{a}}$ & $3,17 \pm 0,02^{d}$ & $1,46 \pm 0,02^{a}$ & $678,06 \pm 0,24^{f}$ & $737,71 \pm 0,23^{d}$ \\
\hline Sapoti & $2,6 \pm 0,02^{i}$ & $0,7 \pm 0,01^{d}$ & $0,53 \pm 0,01^{h}$ & $0,23 \pm 0,02^{g}$ & $1336,71 \pm 0,32^{\mathrm{e}}$ & $1041,56 \pm 0,13^{b}$ \\
\hline Seriguela & $13,3 \pm 0,04^{c}$ & $1,5 \pm 0,01^{b}$ & $1,87 \pm 0,02^{e}$ & $0,66 \pm 0,01^{b}$ & $2418,61 \pm 0,12^{d}$ & $898,74 \pm 0,31^{c}$ \\
\hline Pinha & $13,6 \pm 0,03^{b}$ & $1,3 \pm 0,01^{c}$ & $3,65 \pm 0,02^{b}$ & $0,49 \pm 0,01^{d}$ & $4753,60 \pm 0,53^{b}$ & $571,28 \pm 0,18^{f}$ \\
\hline
\end{tabular}

Dados apresentados como média \pm desvio padrão de três replicatas/amostra. Valores da mesma coluna que apresentam a mesma letra não apresentam diferenças significativas $(p>0,05)$ pelo Teste de Scott-Knotta 95\% de confiança.

É importante destacar que as cascas de frutas são ricas em antocianinas, podendo assim tornar-se matérias-primas importantes para a extração deste pigmento natural para uso em indústria de alimentos, farmacêutica e cosmética. ${ }^{61}$ Atualmente, diferentes estudos científicos e tecnológicos têm evidenciando processos inovadores para a obtenção de antocianinas utilizando subprodutos da industrialização de frutas, como por exemplo, as cascas de uva. ${ }^{21,62}$

Os teores de compostos fenólicos nas polpas das frutas avaliadas variaram entre 69,60 a $1300,18 \mathrm{mg} E A G / 100 \mathrm{~g}$, enquanto na casca essa variação ocorreu entre 191,10 a 5506,78 mg EAG/100 g (Tabela 3). Dentre as frutas analisadas, $90 \%$ apresentaram 0 quantitativo de compostos fenólicos totais na casca superior ao encontrado na polpa. Assim, apenas a mangaba apresentou o teor de fenólicos inferior na casca $(678,06 \mathrm{mg}$ $E A G / 100 \mathrm{~g})$ em relação à polpa $(737,71 \mathrm{mg}$ EAG/100 g). Para a atemoia os resultados estão semelhantes aos valores identificados por Cruz et al. (2013), ${ }^{36}$ que também verificaram que os compostos fenólicos estão em menor concentração na polpa. Na porção comestível da graviola foi verificado valor superior da mesma classe de compostos ao observado por Kuskoski et al. (2005), ${ }^{63}$ de 84,3 mg EAG/100 g.

Faller \& Fialho $(2009)^{64}$ e Lima et al. $(2013)^{65}$ observaram que frutos do cerrado apresentam teores de compostos fenólico 
totais semelhantes com frutas comerciais como manga, abacaxi, mamão, laranja, tangerina e banana. Em estudo realizado por Vieira et al. (2011) ${ }^{66}$ foi verificado variações consideráveis de compostos fenólicos em polpas de frutas congeladas (acerola, caju, goiaba, bacuri, tamarindo).

Uma diversidade de estudos comprova que as frutas são ricas em muitos nutrientes e compostos antioxidantes, e que esses constituintes se concentram majoritariamente nas cascas ou sementes. ${ }^{67,68,69,70,71}$ As frutas processadas, como as bebidas de frutas são uma importante fonte de compostos bioativos, vitaminas e minerais, e, seus extratos merecem atenção especial por causa da influência positiva que podem promover para a saúde do consumidor. ${ }^{72,73}$ Dantas et al. $(2014)^{74}$ evidenciaram a característica antioxidante de algumas frutas tropicais ao incorporar os extratos obtidos em embalagens biodegradáveis, evitando assim a oxidação do produto embalado. Dessa forma, foi evidenciada outra importante aplicação tecnológica das frutas.

\section{Conclusão}

As cascas da maioria das frutas apresentam uma maior concentração dos compostos avaliados, entre eles, os compostos fitoquímicos (fenólicos, flavonoides e antocianinas), demonstrando o potencial de aplicabilidade destas matrizes em diferentes setores industriais, como a indústria de alimentos, farmacêutica e cosmética.

Observa-se os maiores teores de proteína na polpa de jaca, cajarana sapoti e fruta-pão, enquanto que nas outras frutas o maior percentual desse parâmetro foi identificado nas cascas.

O potássio é o mineral de maior expressividade encontrado nas amostras avaliadas, tendo maior porção na casca das frutas, exceto na seriguela.

Partes intituladas comumente como "não comestíveis" nas frutas possuem alguns nutrientes que são fisiologicamente importantes e em quantidades maiores que nas partes "comestíveis" (polpas), evidenciando seu potencial de aplicação em diferentes áreas industriais (alimentícia, farmacêutica e cosmética).

\section{Agradecimentos}

Ao Serviço Nacional de Aprendizagem Industrial - SENAI CIMATEC pela infraestrutura e financiamento para a realização da pesquisa.

\section{Referências Bibliográficas}

${ }^{1}$ Lopes, R. M.; Silva, J. P.; Vieira, R. F.; Silva, D B.; Gomes, I. S.; Agostini-Costa, T. S. Composição de ácidos graxos em polpa de frutas nativas do cerrado. Revista Brasileira de Fruticultura 2012, 34, 635. [Link]

${ }^{2}$ Malta; L. G.; Tessaro, E. P.; Eberlin, M.; Pastore, G.M; Liu; R.H. Assessment of antioxidant and antiproliferative activities and the identification of phenolic compounds of exotic Brazilian fruits. Food Research International 2013, 53, 417. [CrossRef]

${ }^{3}$ Morzelle, M. C.; Bachiega, P.; de Souza, E. C.; Vilas Boas, E. V. B.; Lamounier, M.L. Caracterização química e física de frutos de curriola, gabiroba e murici provenientes do cerrado brasileiro. Revista Brasileira de Fruticultura 2015, 37, 96. [CrossRef]

${ }^{4}$ Bataglion, G. A.; Silva, F. M. A.; Eberlin, M. N.; Koolen, H. H. F. Determination of the phenolic composition from Brazilian tropical fruits by UHPLC-MS/MS. Food Chemistry 2015, 180, 280. [CrossRef]

${ }^{5}$ IBRAF - Instituto Brasileiro de Frutas. Panorama da Cadeia Produtiva das Frutas em 2012 e projeções para 2013. Instituto Brasileiro de Frutas, 2013. [Link]

${ }^{6}$ Septembre-Malaterre, A.; Stanislas, G.; Douraguia, E.; Gonthier, M.P. Evaluation of nutritional and antioxidant properties of the tropical fruits banana, litchi, mango, papaya, 
passion fruit and pineapple cultivated in Réunion French Island. Food Chemistry 2016, 212, 225. [CrossRef]

${ }^{7}$ Lazarini, J. G.; Infante, M. F. J.; Paschoal, J. A. R.; Freires, I. A.; Alencar, S. M.; Rosalen, P. L. Anti-inflammatory activity and polyphenolic profile of the hydroalcoholic seed extract of Eugenia leitonii, an unexplored Brazilian native fruit. Journal of Functional Foods 2016, 26, 249. [CrossRef]

${ }^{8}$ Siqueira, E. M. A.; Rosa, F. R.; Fustinoni, A. M.; Sant'Ana, L. P.; Arruda, S. F. Brazilian savanna fruits contain higher bioactive compounds content and higher antioxidant activity relative tothe conventional red delicious apple. Plos One, 2013, 8, 1. [CrossRef]

${ }^{9}$ Marles, R. J. Mineral nutrient composition of vegetables, fruits and grains: The context of reports of apparent historical declines. Journal of Food Composition and Analysis, 2017, 56, 1. [CrossRef]

10 Lago-Vanzela, E. S; Ramin, P.; Umsza-Guez, M. A.; Santos, G. V; Gomes, E.; Silva, R. Chemical and sensory characteristics of pulp and peel 'cajá-manga' (Spondias cytherea Sonn.) jelly. Ciência e Tecnologia de Alimentos 2011, 31, 398. [CrossRef]

${ }^{11}$ Bueno, S. M.; Lopes, M. R. V.; Graciano, R. A. S.; Fernandes, E. C. B.; Garcia-Cruz, C. H. Avaliação da qualidade de polpas de frutas congeladas. Revista do Instituto Adolfo Lutz 2002, 61, 121. [Link]

12 Abud, A. K. S. E.; Narain, N. Incorporação da farinha de resíduo do processamento de polpa de fruta em biscoitos: uma alternativa de combate ao desperdício. Brazilian Journal of Food Technology 2009, 12. [CrossRef]

13 Oliveira, A. C.; Valentim, I. B.; Silva, C. A., Bechara, E. J. H.; De Barros, M. P.; Mano, C. M.; Goulart, M. O. F. Total phenolic content and free radical scavenging activities of methanolic extract powders of tropical fruit residues, Journal of Agricultural and Food Chemistry 2009, 115, 469. [CrossRef]

14 Balestro, E. A.; Sandri, I. G.; Fontana, R. C. Utilização de bagaço de uva com atividade antioxidante na formulação de barra de cereais. Revista Brasileira de Produtos Agroindustriais 2011, 13, 203. [CrossRef]
${ }^{15}$ Kunradi Vieira, F. G.; Borges, G. S. C.; Copetti, C.; Gonzaga, L. V.; Nunes, E. C.; Fett, R. Activity and contents of polyphenolic antioxidants in the whole fruit, flesh and peel of three apple cultivars. Archivos Latinoamericanos de Nutrición 2009, 59, 101. [PubMed]

${ }^{16}$ Sun, S.; Xin, L.; Gao, H.; Wang, J.; Li, P. Response of phenolic compounds in 'Golden Delicious' and 'Red Delicious' apples peel to fruit bagging and subsequent sunlight reexposure. Scientia Horticulturae 2014, 168, 161. [CrossRef]

17 Toloni, M. H. A.; Longo-Silva, G.; Goulart, R. R. M.; Taddei, J. A. A. C. Introdução de alimentos industrializados e de alimentos de uso tradicional na dieta de crianças de creches públicas no município de São Paulo. Revista de Nutrição, 2011, 24, 61. [CrossRef]

${ }^{18}$ Fonseca, R. S.; Santo, V. R.; Souza, G. B.; Pereira, C. M. A. Elaboração de barra de cereais com casca de abacaxi. Archivos Latinoamericanos de Nutrición 2011, 61, 216. [Link] [PubMed]

${ }^{19}$ Lopes, A.; Sabaini, N.M.; Costa, S.M.G. Produção de biomassa de cogumelo-do-sol e de shitake em resíduos agroindustriais.Boletim do Centro de Pesquisa e Processamento de Alimentos2009, 27, 183. [CrossRef]

${ }^{20}$ Costa, A. B.; De Oliveira, A. M. C.; Silva, A. M. O.; Mancini-Filho, J.; De Lima, A. Atividade antioxidante da polpa, casca e sementes do noni (Morinda citrifolia Linn). Revista Brasileira de Fruticultura 2013, 35, 345. [CrossRef]

${ }^{21}$ Machado, B. A. S.; Silva, C. C.; Guedes, C. M. C.; Umsza-Guez, M. A.; Cirqueira, M. G.; Oliveira, R. S.; INPI 2014. (BR 1020140302425).

22 PLANO NACIONAL DE RESÍDUOS SÓLIDOS. Versão Preliminar para Consulta Pública. Ministério do Meio Ambiente. Governo Federal. Brasília, 2011. [Link]

${ }^{23}$ Sousa, E. P.; Figueirêdo, R. M. F.; Queiroz, A. J. M.; Silva, L. M. M.; Sousa; F. C. Caracterização físico-química da polpa de sapoti oriunda do estado do Ceará. Revista Verde de Agroecologia e Desenvolvimento Sustentável 2012, 7, 45. [Link] 
${ }^{24}$ ASSOCIATION OF OFFICIAL ANALYTICAL CHEMISTS (AOAC). Official methods of analysis. 16nd ed. Arlington, 1997.

${ }^{25}$ Silva, P. H. F.; Carvalho, M. C. L. Determinação de nitrogênio em leite pelo método de Kjeldahl. Revista Instituto de Laticínios Cândido Tostes 1993, 48, 30.

${ }^{26}$ IUPAC - International Union of Pure and Applied Chemistry. Standard methods for the analysis of oils, fats and derivatives, 6th ed., Pergamon Press: Oxford, 1979.

${ }^{27}$ IAL - Instituto Adolfo Lutz. Normas Analíticas do Instituto Adolfo Lutz. Métodos químicos e físicos para análise de alimentos, 4. ed. São Paulo: IMESP, p.77-78; 726-728, 2004. ${ }^{28}$ Larrauri, J. A.; Rupérez, P.; Calixto, F. S. Effect of drying temperature on the stability of polyphenols and antioxidant activity of red grape pomace peels. Journal of Agricultural and Food Chemistry 1997, 45, 1390. [CrossRef] ${ }^{29}$ Obanda, M.; Owuor, P. O. Flavanol Composition and caffeine content of green leaf as quality potential indicators of Kenyan black teas. Journal of the Science of Food and Agriculture 1997, 74, 209. [CrossRef]

${ }^{30}$ Francis, F. J. Analysis of anthocyanins, In: MARKAKIS, P. Anthocyanins as Food Colors. New York: Academic Press, p. 182-205, 1982.

${ }^{31}$ Canuto, G. A. B.; Xavier, A. A. O.; Neves, L. C.; Benassi, M. T. Caracterização físicoquímica de polpas de frutos da Amazônia e sua correlação com a atividade anti-radical livre. Revista Brasileira de Fruticultura 2010, 32, 1196. [CrossRef]

${ }^{32}$ Andrade, S. A. C.; Neto, B. B.; Salgado, S. M.; Guerra, N. B. Influência de revestimentos comestíveis na redução de ganho de sólidos em jenipapos desidratados osmoticamente. Ciência e Tecnologia de Alimentos 2007, 27, 39. [CrossRef]

${ }^{33}$ Khakimov, B., Mongia, R. J.; Sørensen, K. M.; Ndabikunze, B. K.; Chove, B. E.; Engelsen, S. B. A comprehensive and comparative GC-MS metabolomics study of non-volatiles in Tanzanian grown mango, pineapple, jackfruit, baobab and tamarind fruits. Food Chemistry 2016, 213, 691. [CrossRef]

${ }^{34}$ Marcellini, P. S; Cordeiro, C. E.; Faraoni, A. S.; Batista, R.A.; Ramos, A. L. D.; Lima, Á. S. Comparação físico-química e sensorial da atemoia com a pinha e a graviola produzidas e comercializadas no estado de Sergipe. Alimento e Nutrição 2003, 14, 187. [Link]

${ }^{35}$ Lima, I. C. G. S.; Dissertação de Mestrado, Universidade Federal Rural do Rio de Janeiro, 2009.

${ }^{36}$ Cruz, L. S.; Lima, R. Z.; Abreu, C. M. P.; Corrêa, A. D.; Pinto, L. A. Caracterização física e química das frações do fruto atemoia Gefner. Ciência Rural 2013, 43, 2280. [Link]

${ }^{37}$ Monteiro, B. A.; Dissertação de Mestrado, Faculdade Estadual Paulista, 2009.

${ }^{38}$ Silva, M. R; Lacerda, D. B. C. L.; Santos, G. G; Martins, D. M. O. Caracterização química de frutos nativos do cerrado. Ciência Rural 2008, 38, 1790. [CrossRef]

${ }^{39}$ TACO - Tabela Brasileira de Composição de Alimentos. Núcleo de Estudos e Pesquisas em Alimentação - NEPA. Universidade Estadual de Campinas - UNICAMP. Versão 2, 2ª Edição. Campinas - SP, 2006.

${ }^{40}$ Souza, D. S.; Souza, J. D. R. P; Coutinho, J. P.; Ferrão, S. P. B; Souza, T. S.; Silva, A. A. L. Elaboração de farinha instantânea a partir da polpa de fruta-pão (Artocarpus altilis). Ciência Rural 2012, 42, 1123. [Link]

${ }^{41}$ Motta, E. L.; Dissertação de Mestrado, Universidade Federal do Rio de Janeiro, 2009.

42 Rizzon, L. A.; Bernardi, J.; Miele, A. Características analíticas dos sucos de maçã gala, Golden Delicious e Fuji. Ciência $e$ Tecnologia de Alimentos 2005, 25, 750. [CrossRef]

${ }^{43}$ Andrade, E. C. B.; Barros, A. M.; Takase, I. Avaliação da solubilidade de cobre e zinco em caldos de leguminosas. Ciência e Tecnologia de Alimentos 2003, 3, 386. [CrossRef]

${ }^{44} \mathrm{VI}$ DIRETRIZES BRASILEIRAS DE HIPERTENSÃO. Arquivos Brasileiros de Cardiologia2010, 1, 17. [CrossRef] [Link]

${ }^{45}$ Bhat, R.; Paliyath, G. Fruits of Tropical Climates: Biodiversity and Dietary Importance. Reference Module in Food Science, from Encyclopedia of Food and Health, p.138-143, 2016.

${ }^{46}$ Morais, D. R.; Rotta, E. M.; Sargi, S. C.; Schmidt, E. M.; Bonafe, E. G.; Eberlin, M. N.; Sawaya, A. C. H. F.; Visentainer, J. V. Antioxidant activity, phenolics and UPLC-ESII)-MS of extracts from different tropical fruits parts and processed peels. Food Research International 2015, 77, 392. [CrossRef] 
47 Uchôa, A. M. A.; Dissertação de Mestrado, Universidade Federal do Ceará, 2007.

${ }^{48}$ Ribeiro, R. D.; Finzer, J. R. D. Desenvolvimento de biscoito tipo cookie com aproveitamento de farinha de sabugo de milho e casca de banana. Faculdades Associadas de Uberaba. FAZU 2010, 7, 120. [Link]

${ }^{49}$ Santana, F. C.; Silva, J. V.; Santos, A. J. A. O.; Alves, A. R; Wartha, E. R. S. A; Marcellini, P. S.; Silva, M. A. A. P. Desenvolvimento de biscoito rico em fibras elaborado por substituição parcial da farinha de trigo por farinha da casca do maracujá amarelo (Passiflora edulis flavicarpa) e fécula de mandioca (Manihot Esculenta crantz). Alimentos e Nutrição 2011, 22, 391. [Link]

50 Damiani, C.; Boas, E. V. B. V.; Júnior, M. S. S.; Caliari, M.; Paula, M. L.; Pereira, D. E. P.; Silva, A. G. M. Análise física, sensorial e microbiológica de geléias de manga formuladas com diferentes níveis de cascas em substituição à polpa. Ciência Rural 2008, 38, 1418. [CrossRef]

${ }^{51}$ Ruviaro, L.; Novello, D.; Almeida, J. M.; Quintiliano, D. A. Análise sensorial de sobremesa acrescida a farelo de casca e bagaço de laranja entre universitários de Guarapuava (PR). Revista Salus 2008, 2, 42. [Link]

52 Vicenzi, R.; Bilhalva, A. B; Treptow, R. O. Avaliação sensorial do suco de maçã processado com casca de arroz como coadjuvante de prensagem. Ciência $e$ Tecnologia de Alimentos 2001, 21, 257. [Link]

${ }^{53}$ Fernandes, A. F.; Dissertação de Mestrado, Universidade Federal de Lavras, 2006.

54 Bolanho, B. C.; Danesi, E. D. G.; Beléia, A. P. Characterization of flours made from peach palm (Bactris gasipaes Kunth) by-products as a new food ingredient. Journal of Food and Nutrition Research 2014, 53, 51. [Link]

55 Roesler, R.; Malta, L. G; Carrasco, L. C.; Holanda, R. B.; Sousa, C. A. S; Pastore, G. M. Atividade antioxidante de frutas do cerrado. Ciência e Tecnologia de Alimentos 2007, 27, 53. [CrossRef]

56 Morais, M. L.; Silva, A. C. R.; Araújo, C. R. R.; Esteves, E. A., Dessimoni-Pinto, N. A. V. Determinação do potencial antioxidante in vitro de frutos do cerrado brasileiro. Revista Brasileira de Fruticultura 2013, 35, 355. [CrossRef]

57 Rocha, M. S.; De Figueiredo, R. W.; Araújo, M. A. M.; Moreira-Araújo, R. S. R. Caracterização físico-química e atividade antioxidante (in vitro) de frutos do cerrado piauiense. Revista Brasileira de Fruticultura 2015, 37, 96. [CrossRef]

58 Huber, L. S.; Rodriguez-Amaya, D. B. Flavonóis e flavonas: fontes brasileiras e fatores que influenciam a composição em alimentos. Alimento e Nutrição 2008, 19, 97. [Link]

${ }^{59}$ Março, P. H.; Scarminio, I. S. Procedimentos analíticos para identificação de antocianinas presentes em extratos naturais. Química Nova 2008, 31, 1218. [CrossRef]

${ }^{60}$ Favaro, M.M.A.; Dissertação de Mestrado, Universidade Estadual de Campinas, 2008.

${ }^{61}$ Falcão, A. P.; Chaves, E. S.; Kuskoski, E. M.; Fett, R.; Falcão, L. D.; Bordignon-Luiz, M. T. Índice de polifenóis, antocianinas totais e atividade antioxidante de um sistema modelo de geleia de uvas. Ciência e Tecnologia de Alimentos 2007, 27, 637. [CrossRef]

${ }^{62}$ Amorim, F. L.; Rocha. I. S.; Ferreira, E. S.; Machado, B. A. S.; Umsza-Guez, M. A. Prospecção tecnológica relativa a depósitos de patentes relacionadas aos compostos bioativos presente em uvas. Caderno de Prospecção 2015, 8, 797. [CrossRef]

${ }^{63}$ Kuskoski, E. M.; Asuero, A. G.; Troncoso, A. M.; Mancini-Filho, J.; Fett, R. Aplicación de diversos métodos químicos para determinar actividad antioxidante en pulpa de frutos. Ciência e Tecnologia de Alimentos 2005, 25, 726. [CrossRef]

${ }^{64}$ Faller, A. L. K.; Fialho, E. Disponibilidade de polifenóis em frutas e hortaliças consumidas no Brasil. Revista de Saúde Pública 2009, 43, 211. [CrossRef]

${ }^{65}$ Lima, C. A.; Faleiro, F. G.; Junqueira, N. T. V.; Cohen, K. O.; Guimarães, T. G. Características físico-químicas, polifenóis e flavonoides amarelos em frutos de espécies de pitaias comerciais e nativas do cerrado. Revista Brasileira de Fruticultura 2013, 35, 565. [CrossRef] 
${ }^{66}$ Vieira, L. M.; Sousa, M. S. B.; Mancini-Filho, J.; Lima, A. Fenólicos totais e capacidade antioxidante. Revista Brasileira de Fruticultura 2011, 33, 888. [CrossRef]

${ }^{67}$ Souza, M. S. B.; Vieira, L. M.; Silva, M. J. M.; Lima, A. Caracterização nutricional e compostos antioxidantes em resíduos de polpas de frutas tropicais. Ciência $e$ agrotecnologia 2011, 35, 554. [CrossRef]

${ }^{68}$ Gondim, J. A. M.; Moura, M. F. V.; Dantas, A. S.; Medeiros, R. L. S.; Santos, K. M. Composição Centesimal e de minerais em cascas de frutas. Ciência e Tecnologia de Alimentos 2005, 25, 825. [Link]

${ }^{69}$ Oliveira, L. F.; Nascimento, M. R. F.; Borges, S. V.; Ribeiro, P. C.; Ruback, V. R. Aproveitamento alternativo da casca do maracujá-amarelo (Passiflora edulis F. Flavicarpa) para produção de doce em calda. Ciência e Tecnologia de Alimentos 2002, 22, 259. [Link]

${ }^{70}$ Ferrari, R. A.; Colussi, F.; Ayub, R. A. Caracterização de subprodutos da industrialização do maracujá-aproveitamento das sementes. Revista Brasileira de Fruticultura 2004, 26, 101. [CrossRef]
${ }^{71}$ Borges, T. H.; Rodrigues, N.; Souza, A. M. Effect of different extraction conditions on the antioxidant potential of baru almonds (Dipteryx alataVog.): comparison to common nuts from Brazil. Journal of Food and Nutrition Research 2014, 53, 180. [Link]

${ }^{72}$ Andrés, V.; Villanueva, M. J.; MateosAparicio, I.; Tenorio, M. D. Colour, bioactive compounds and antioxidant capacity of mixed beverages based on fruit juices with milk or soya. Journal of Food and Nutrition Research 2014, 53, 71.

${ }^{73}$ Dimitrijević, D. S.; Kostić, D. A.; Stojanović, G. S.; Mitić, S. S.; Mitić, M. N.; Dordević, A. S. Phenolic composition, antioxidant activity, mineral content and antimicrobial activity of fresh fruit extracts of Morus alba L. Journal of Food and Nutrition Research 2014, 53, 22. [Link]

${ }^{74}$ Dantas, E. A.; Costa, S. S.; Cruz, L. S.; Bramont, W. B.; Costa, A. S.; Padilha, F. F.; Druzian, J. I.; Machado, B. A. S. Caracterização e avaliação das propriedades antioxidantes de filmes biodegradáveis incorporados com polpas de frutas Tropicais. Ciência Rural 2014, 45, 142. [CrossRef] 